\title{
Microneedles 2012
}

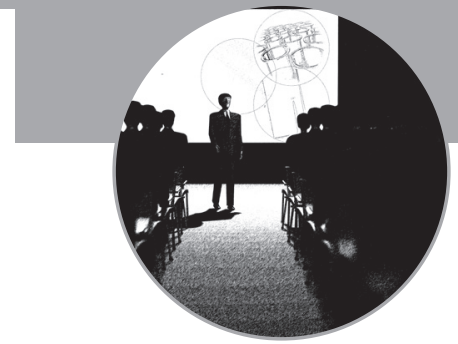

\section{2nd International Conference on Microneedles}

\author{
13-15 May 2012, Cork, Republic of Ireland
}

The second international conference on Microneedles was held on 13-15 May 2012 in Cork, Republic of Ireland, following on from a successful first meeting at the Georgia Institute of Technology (GA, USA) in May 20I0. The meeting showcased the latest international developments in microneedle technology and applications. The gathering provided a platform to facilitate interdisciplinary communications and new collaborations for delegates from academic, industrial and clinical backgrounds. The meeting opened with a half-day short course on microneedle technology and applications, followed by invited lectures and poster presentations over 2 days, divided into sessions such as 'Design and technology - solid and hollow microneedles', 'Vaccine delivery' and 'Drug delivery'. This conference report summarizes the keynote and invited speaker lectures from leaders in the field such as Mark Prausnitz (Georgia Institute of Technology) and Mark Kendall (University of Queensland, Australia).

The second international conference on Microneedles was hosted by the Tyndall National Institute and University College Cork, and was held in Cork in the south of the Republic of Ireland. The conference was attended by 185 delegates from over 24 countries including the USA, Australia, South Korea, Germany, the UK and Ireland. Since microneedle $(\mathrm{MN})$ research is a highly multidisciplinary area, the meeting drew input from biomedical, scientific and engineering disciplines, with specific emphasis on relevant pharmaceutical, clinical and regulatory topics. The Microneedles 2012 meeting fostered an exchange of ideas in a relaxed and informal setting for delegates from academic, industrial and clinical backgrounds. Representatives from 58 companies were present and the split of delegates was almost equal between academia (56\%) and industry (44\%).

The first day opened with a session titled 'Design and technology - solid and hollow microneedles'. The keynote speaker for this session was Mark Prausnitz of the Georgia Institute of Technology (GA, USA), whose talk was titled 'Evaluation of microneedles in human subjects'. Prausnitz's talk was divided into four sections covering skin pretreatment, coated MNs, hollow $\mathrm{MNs}$ and patient acceptance. Initially, we saw data on the kinetics of skin resealing following $\mathrm{MN}$ insertion. This varied considerably if the skin was occluded or not, with the barrier function being restored quicker without coverage. We were then shown clinical data concerning the delivery of parathyroid hormone administration in postmenopausal women for the treatment of osteoporosis through a coated MN patch. Data were also shown for hollow MN delivery where insertion depth and fluid flow rate was investigated and correlated to visual analogue scale pain scores [1]. This technology was also used for the delivery of lidocaine and insulin. Finally, the talk was concluded with a section on patient acceptability. The Prausnitz group had investigated whether their patch technology was considered more acceptable from a patient perspective in delivering the seasonal influenza shot in comparison to standard intramuscular (im.) delivery. Since pain can be minimized or eliminated by $\mathrm{MN}$ delivery, the patch was actively preferred over the im. delivery.

The second session of the day was entitled 'Design and technology - polymer and biodegradable microneedles'. The first invited speaker for the session was Kris Hansen from 3M Drug Delivery Systems (MN, USA), who spoke about MN-based drug delivery and how less can be more. Hansen introduced 3MIMs MN technology platforms [2]; describing both their hollow and coated technologies and the new microchannel skin system. She described that while MN could certainly be used for vaccine delivery, another application might be the improvement in delivery of therapeutics for chronic conditions that currently require multiple daily or weekly injections. Hansen described clinical data from a collaboration with Radius Health (MA, USA) looking at the delivery of their osteoporosis drug in a patch formulation.

The second invited speaker in this session was Jung-Hwan Park from Gachon

\section{Kate E Broderick}

Inovio Pharmaceuticals, Inc., II494 Sorrento Valley Road Suite A, San Diego, CA, USA Tel.: +| 8584103161 Fax: +| 858597045 | E-mail: kbroderick@inovio.com 
University (Korea). He spoke about biodegradable polymer $\mathrm{MN}$ separation in skin, mediated by hydrogel swelling [3]. He described how hydrogel needles had benefits over standard biodegradable needles, in that the thermal processing that can damage protein biologics is not required, and that there is very efficient tip separation.

The afternoon session was entitled 'Coatings and formulations', and the keynote speaker was Mark Kendall from the University of Queensland (Australia). Kendall initially gave an introduction to vaccine delivery, and coating and release technologies, as well as delivery of vaccines to the skin. He then described several in vivo immunogenicity studies in mice where dose-sparing regimes were investigated. Data described during the talk demonstrated that equivalent humoral and T-cell responses were generated using the Nanopatch technology with the vaccines delivered at dosesparing levels over a full dose delivered in a standard manner. Kendall ended by describing a possible alterative use for the patch technology for high throughput disease diagnosis. He showed a data slide on a dengue mouse model [4].

The second invited speaker of that session was Ronald Pettis from BD technologies, NC, USA. Pettis described the use of MN for lymphatic targeting [5]. We observed data from a mouse melanoma study delivering IL-12 and saw that the MN route had consistent affect on reducing tumor area; whereas, the intraperitoneal and subcutaneous routes did not. There was also a significant increase in NK cells in lymph nodes following MN delivery of IL-12; whereas, no increases were seen in intraperitoneal and subcutaneous groups. Pettis also described delivery of antibodies via this route and again showed improved tumor reduction and reduced tumor vessel density with $\mathrm{MN}$ delivery.

The second day opened with a session titled 'Vaccine delivery'. The keynote speaker was Joke Bouwstra from the Leiden/Amsterdam Center for Drug Research (The Netherlands) [6]. Bouwstra opened by describing the barrier properties of the stratum corneum and suggested that you can reduce this barrier function through the pretreatment of skin with MN. The effect of $\mathrm{MN}$ pretreatment on immune responses was described in a mouse model, and the data suggested that the MN caused a dramatic increase in humoral responses following the application of diphtheria toxin to pre-treated skin. Bouwstra also discussed the use of nanoparticles for enhanced delivery to the skin. The nanoparticles used in this study were trimethyl chitosan and she demonstrated that delivery via trimethyl chitosan caused increased uptake to dendritic cells and improved dendritic cell maturation.

The invited speaker for this session was Yotam Levin from NanoPass, Israel. Levin spoke about NanoPass novel MN device for intradermal delivery and presented clinical experience and commercial opportunities. The talk was opened with a description of the MicronJet device and how it was the first MN device to secure US FDA regulation (510k approval), CE marking and also ISO13485 certification. The device is single use, simple to use and considered nearly painless. Levin then detailed multiple clinical studies where the MicronJet device had been used in comparison to standard im. delivery of vaccine and showed the benefit of this device for dose sparing [7]. In particular, one Phase II study from 2007/2008 compared a $3 \mu \mathrm{g}$ dose of standard influenza vaccine delivered via the MicronJet with $15 \mu \mathrm{g}$ dose delivered via im. injection and 3, 4.5 and $5 \mu \mathrm{g}$ doses delivered via intradermal injection. The hemagluttanin inhibition assay and seroconversion data were equivalent across groups. The device was seen to induce mild erythema in subjects but was considered highly tolerated and generally preferred over the im. route.

In the second morning session also titled 'Vaccine delivery', the invited speaker was Kate Broderick from Inovio Pharmaceuticals (CA, USA). Broderick spoke about Inovio's dermal electroporation (EP) technology and how this would be applicable to DNA vaccine delivery via MNs. She introduced the dermal EP technology, describing the minimally invasive device, the CELLECTRA-3P ${ }^{\circledR}$ and described the ongoing clinical trial using this technology. The rest of the talk detailed the next-generation surface $[8]$ and contactless EP devices, and showed localization and immunogenicity data, as well as describing how this technology could be applied to direct mucosal immunization.

The afternoon sessions covering drug delivery via $\mathrm{MNs}$ and clinical applications of $\mathrm{MN}$ technology are not detailed in this report.

\section{Summary}

Conor O'Mahony, Chair of the Microneedle 2012 organizing committee, concluded the meeting by summarizing the topics covered in the preceding days and thanking all the speakers, poster presenters and attendees for participating in a stimulating and informative forum. Based on the overwhelming success of this meeting and the previous gathering in Georgia in 2010, there was additional discussion as to the venue for the 2014 3rd International Conference on Microneedles. 


\section{Financial \& competing interests disclosure} $K E$ Broderick has financial involvements with Inovio Pharmaceuticals through employment, and stock ownership and options. The author has no other relevant affiliations or financial involvement with any organization or entity with a financial interest in or financial conflict with the subject matter or materials discussed in the manuscript apart from those disclosed.

No writing assistance was utilized in the production of this manuscript.

\section{References}

1 Gupta J, Park SS, Bondy B, Felner EI, Prausnitz MR. Infusion pressure and pain during microneedle injection into skin of human subjects. Biomaterials 32(28), 6823-6831 (2011).

2 Duan D, Moeckly C, Gysbers J et al. Enhanced delivery of topically-applied formulations following skin pre-treatment with a hand-applied, plastic microneedle array. Curr. Drug Deliv. 8(5), 557-565 (2011).

3 Kim M, Jung B, Park JH. Hydrogel swelling as a trigger to release biodegradable polymer microneedles in skin. Biomaterials 33(2), 668-678 (2012).
4 Muller DA, Corrie SR, Coffey J, Young PR, Kendall MA. Surface modified microprojection arrays for the selective extraction of the dengue virus NS1 protein as a marker for disease. Anal. Chem. 84(7), 3262-3268 (2012).

5 Harvey AJ, Kaestner SA, Sutter DE, Harvey NG, Mikszta JA, Pettis RJ. Microneedle-based intradermal delivery enables rapid lymphatic uptake and distribution of protein drugs. Pharm. Res. 28(1), 107-116 (2011).

6 van der Maaden K, Jiskoot W, Bouwstra J. Microneedle technologies for (trans) dermal drug and vaccine delivery. J. Control. Release 161(2), 645-655 (2012).

7 Van Damme P, Oosterhuis-Kafeja F, Van der Wielen M, Almagor Y, Sharon O, Levin Y. Safety and efficacy of a novel microneedle device for dose sparing intradermal influenza vaccination in healthy adults. Vaccine 27(3), 454-459 (2008)

8 Broderick KE, Shen X, Soderholm J et al. Prototype development and preclinical immunogenicity analysis of a novel minimally invasive electroporation device. Gene Ther. 18(3), 258-265 (2011). 